\title{
Data Homogeneity Effect in Deep Learning-Based Prediction of Type 1 Diabetic Retinopathy
}

\author{
Jui-En Lo $\mathbb{D}^{1,},{ }^{1,2}$ Eugene Yu-Chuan Kang $\mathbb{D}^{3,4,5}$ Yun-Nung Chen $\mathbb{D}^{2}{ }^{2}$ Yi-Ting Hsieh $(\mathbb{D})^{6}$

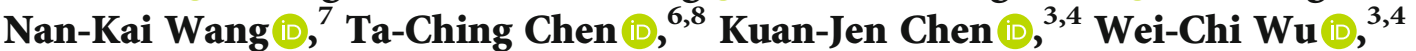 \\ Yih-Shiou Hwang $\mathbb{D}^{3,4,9,10}$ Fu-Sung Lo $\mathbb{D}^{4,11}$ and Chi-Chun Lai $\mathbb{D}^{3,4,12}$
}

${ }^{1}$ School of Medicine, National Taiwan University College of Medicine, Taipei 106, Taiwan

${ }^{2}$ Department of Computer Science and Information Engineering National Taiwan University, Taipei 106, Taiwan

${ }^{3}$ Department of Ophthalmology, Chang Gung Memorial Hospital, Linkou Medical Center, Taoyuan 333, Taiwan

${ }^{4}$ College of Medicine, Chang Gung University, Taoyuan 333, Taiwan

${ }^{5}$ Graduate Institute of Clinical Medical Sciences, Chang Gung University, Taoyuan 333, Taiwan

${ }^{6}$ Department of Ophthalmology, National Taiwan University Hospital, Taipei 100, Taiwan

${ }^{7}$ Department of Ophthalmology, Edward S. Harkness Eye Institute, Columbia University, New York, New York 10032, USA

${ }^{8}$ Graduate Institute of Clinical Medicine, College of Medicine, National Taiwan University, Taipei 106, Taiwan

${ }^{9}$ Department of Ophthalmology, Chang Gung Memorial Hospital, Xiamen 361028, China

${ }^{10}$ Department of Ophthalmology, Jen-Ai Hospital Dali Branch, Taichung 400, Taiwan

${ }^{11}$ Division of Pediatric Endocrinology and Genetics, Chang Gung Memorial Hospital, Linkou Medical Center, Taoyuan 333, Taiwan

${ }^{12}$ Department of Ophthalmology, Chang Gung Memorial Hospital, Keelung 204, Taiwan

Correspondence should be addressed to Fu-Sung Lo; lofusu@cgmh.org.tw and Chi-Chun Lai; chichun.lai@gmail.com

Jui-En Lo and Eugene Yu-Chuan Kang contributed equally to this work.

Received 1 September 2021; Revised 27 October 2021; Accepted 25 November 2021; Published 28 December 2021

Academic Editor: Tao Li

Copyright (C) 2021 Jui-En Lo et al. This is an open access article distributed under the Creative Commons Attribution License, which permits unrestricted use, distribution, and reproduction in any medium, provided the original work is properly cited.

This study is aimed at evaluating a deep transfer learning-based model for identifying diabetic retinopathy (DR) that was trained using a dataset with high variability and predominant type 2 diabetes (T2D) and comparing model performance with that in patients with type 1 diabetes (T1D). The Kaggle dataset, which is a publicly available dataset, was divided into training and testing Kaggle datasets. In the comparison dataset, we collected retinal fundus images of T1D patients at Chang Gung Memorial Hospital in Taiwan from 2013 to 2020, and the images were divided into training and testing T1D datasets. The model was developed using 4 different convolutional neural networks (Inception-V3, DenseNet-121, VGG1, and Xception). The model performance in predicting DR was evaluated using testing images from each dataset, and area under the curve (AUC), sensitivity, and specificity were calculated. The model trained using the Kaggle dataset had an average (range) AUC of 0.74 (0.03) and $0.87(0.01)$ in the testing Kaggle and T1D datasets, respectively. The model trained using the T1D dataset had an AUC of 0.88 (0.03), which decreased to 0.57 (0.02) in the testing Kaggle dataset. Heatmaps showed that the model focused on retinal hemorrhage, vessels, and exudation to predict DR. In wrong prediction images, artifacts and low-image quality affected model performance. The model developed with the high variability and T2D predominant dataset could be applied to T1D patients. Dataset homogeneity could affect the performance, trainability, and generalization of the model. 


\section{Introduction}

Diabetic retinopathy (DR) is a severe vascular complication that may lead to blindness in patients with type 1 diabetes (T1D) [1]. As early detection and intervention can delay disease progression, patients are encouraged to undergo eye examination 3-5 years after the onset of disease and an annual DR screening thereafter [2, 3]. Despite the benefits of early treatment, approximately $60 \%$ of patients receive regular DR screening [2]. Reported reasons for nonadherence to recommended annual screening include cost, lack of access to eye care, and no perceived need [4]. Therefore, automated detection may fill these resource gaps and even improve patient outcomes by providing timely detection.

In an era of advancing technology in artificial intelligence, numerous studies have proven the effectiveness of applying deep convolutional networks for detecting DR [5-7]. However, wide variability exists in the approaches to prediction problems across different studies [8]. Understanding the factors that influence the reliability and robustness of the algorithm is important for clinical deployment and may help ensure consistency in performance under various conditions. A previous study reviewed the potential factors [9]; however, whether different etiologies of diabetes or variability of training images affect the algorithm's performance has yet to be investigated.

Diabetes patients can be classified into two major categories based on different etiologies: T1D, which is caused by insulin deficiency and is also known as insulin-dependent diabetes, and type 2 diabetes (T2D), which is caused by insulin resistance and is also known as insulin-independent diabetes mellitus. T2D accounts for most cases of diabetes. In the US population, $>90 \%$ of patients with diabetes have T2D, whereas T1D accounts for only 5\% [10]. T1D predominantly affects the European population [11], and its prevalence in the Asian population is even lower; for example, T1D is present in $<1 \%$ of the diabetic population in Taiwan [12]. Although T1D presents as the minority in the diabetic population, patients with T1D are more likely to develop DR and have more severe visual outcomes than patients with T2D $[1,2,13,14]$. A study reported that youth with T1D also develop DR faster than those with T2D [15]. When evaluating the cause of vision impairment in diabetes, DR accounts for $86 \%$ of poor visual acuity in T1D and only $33 \%$ in T2D [14]. As machine learning has been widely used for the automatic detection of DR, most images are obtained from the dataset predominantly containing the images of T2D patients. Furthermore, the investigation of the performance of models in identifying DR in the specific T1D population is limited.

To assess whether different etiologies of diabetes (i.e., T1D and T2D) affect the performance and robustness of deep learning models, we conducted this study using deep learning models trained using two datasets: one from open-access datasets with high image variability from T2D patients predominantly and the other one consisting of images obtained only from T1D patients followed at a single medical center. As our dataset is small compared with the recommended size [9], the deep transfer learning method is preferably used, which allows for low training cost and the use of a smaller training dataset by reusing a pretrained network to solve a different task [16]. The performance and heatmaps of deep learning models trained with the two datasets were then compared.

\section{Materials and Methods}

2.1. Datasets. An open-access dataset was subsampled from one of the Kaggle datasets, namely, Train.001, which is a publicly available dataset provided by EyePACS [17], which contained a group of patients with a mean age of around 55.4 years and a standard deviation of 11.3 years [5]. The other dataset of retinal fundus images was retrospectively acquired from T1D patients at a 3700-bed medical center, Chang Gung Memorial Hospital, Linkou Medical Center, Taiwan, between 2013 and 2020. All T1D patients were from the Chang Gung Juvenile Diabetes Eye Study [18, 19] and diagnosed based on the World Health Organization diagnosis criteria [20]. The T1D dataset consisted of patients with a mean age of 25.7 years and a standard deviation of 5.8 years. In the T1D dataset, two types of color fundus cameras were used (Topcon Medical Systems, Oakland, NJ, USA; Kowa, Tokyo, Japan, and Digital Non-Mydriatic Retinal Camera, Canon, Tokyo, Japan). Image resolution in both datasets ranged from $1,000 \times 1,500$ to $2,500 \times 3,500$ pixels. This study was approved by the Institutional Review Board of Chang Gung Memorial Hospital (no. 201900477B0) and adhered to the tenets of the Declaration of Helsinki.

2.2. Classification of $D R$. Retinal fundus images from T1D patients were graded by two trained retinal ophthalmologists (EYK and NKW) according to the International Clinical Diabetic Retinopathy Disease Severity Scale. Images with artifacts, shadows, or poor quality that could not be classified were excluded. Retinal ophthalmologists were unaware of clinical information, such as demographics, laboratory data, and prior treatment. On the other hand, DR classification in the Kaggle dataset was defined according to the labels provided with the dataset. In this study, DR was defined as the diagnosis of DR at any stage [21].

2.3. Data Preprocessing and Division. All input images from both datasets were cropped and resized using OpenCVpython to a 320-pixel wide square that tightly contained the circular fundus region. Monochromatic fundus photography and images not having both the optic disc and macular region were filtered out (Figure 1). For each dataset, images were randomly divided into two sets: two-thirds in the training set to develop the model and one-third in the testing set to evaluate model performance (Figure 2). Then, the training set was further divided into two subsets: two-thirds of the training set for optimizing the weights of the network and one-third as the validation set to select hyperparameters for the model. As images from the T1D dataset may come from the same patients, to avoid data leakage, images from same patients were placed in the same sets. After division, the images were randomly shuffled in their own dataset to reduce overfitting and variance before training and were then further batch 


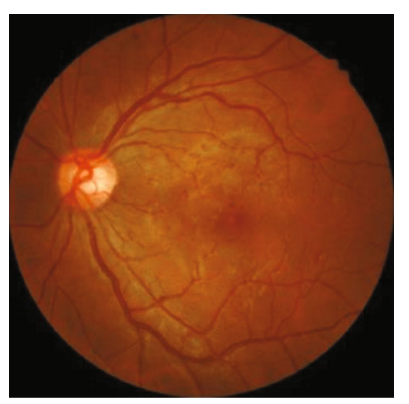

(a)

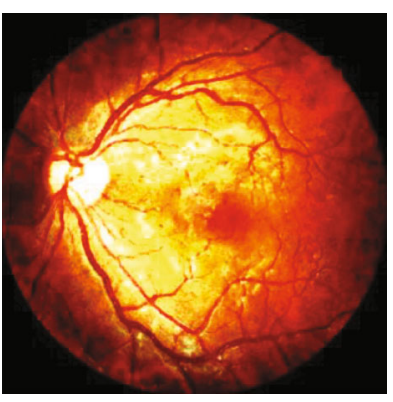

(b)

FIGURE 1: Fundus image after (a) cropping and (b) normalization.

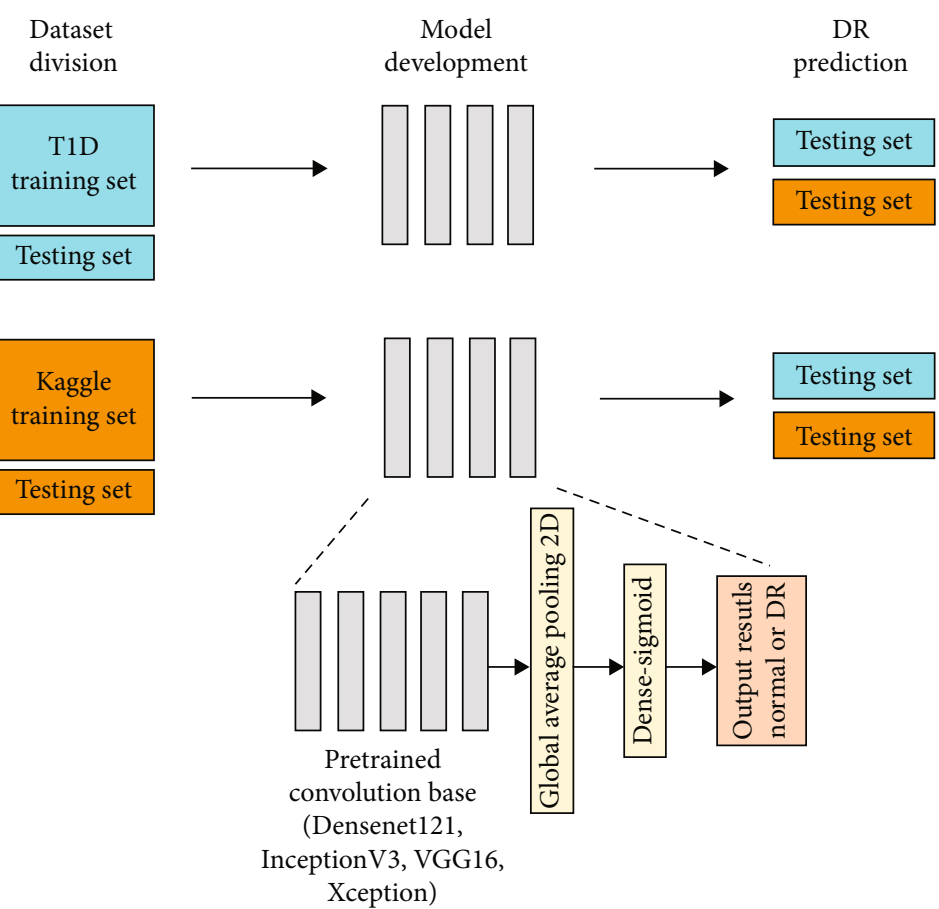

Figure 2: Schematic of the development and evaluation of models. Two groups of models were trained using the T1D and Kaggle training sets, and they were tested with both the T1D and Kaggle testing sets.

TABLE 1: Summary of the prediction performance of different transfer learning models in predicting diabetic retinopathy.

\begin{tabular}{lccccccccccccc}
\hline & \multicolumn{4}{c}{ Trained on Kaggle training set } & \multicolumn{4}{c}{ Trained on T1D training set } \\
& \multicolumn{3}{c}{ Tested on T1D testing } & \multicolumn{3}{c}{ Tested on Kaggle testing } & \multicolumn{3}{c}{ Tested on T1D testing } & \multicolumn{2}{c}{$\begin{array}{c}\text { Tested on Kaggle testing } \\
\text { set }\end{array}$} \\
& AUC & SEN & SPE & AUC & SEN & SPE & AUC & SEN & SPE & AUC & SEN & SPE \\
\hline DenseNet-121 & 0.86 & 0.77 & 0.79 & 0.74 & 0.67 & 0.71 & 0.91 & 0.81 & 0.86 & 0.55 & 0.55 & 0.54 \\
InceptionV3 & 0.86 & 0.74 & 0.79 & 0.74 & 0.62 & 0.74 & 0.87 & 0.73 & 0.86 & 0.59 & 0.56 & 0.59 \\
VGG16 & 0.88 & 0.78 & 0.82 & 0.77 & 0.66 & 0.75 & 0.84 & 0.67 & 0.84 & 0.54 & 0.59 & 0.49 \\
Xception & 0.86 & 0.74 & 0.82 & 0.71 & 0.60 & 0.72 & 0.88 & 0.74 & 0.90 & 0.59 & 0.61 & 0.52 \\
\hline
\end{tabular}

T1D: type 1 diabetes; AUC: area under the curve; SEN: sensitivity; SPE: specificity.

normalized by subtracting the average and dividing by the standard deviation calculated from the training dataset using ImageDataGenerator of Keras API. Real-time data augmentation was applied by randomly rotating, shifting, and shearing the images during the model training based on previously published methods [22].
2.4. Architecture and Evaluation of the Model. The deep transfer learning model consisted of a pretrained convolutional neural network (CNN), followed by a global average pooling layer and a dense layer to output prediction results (Figure 2). The weights from the pretrained model were trainable and were used to extract image features, and predictions 


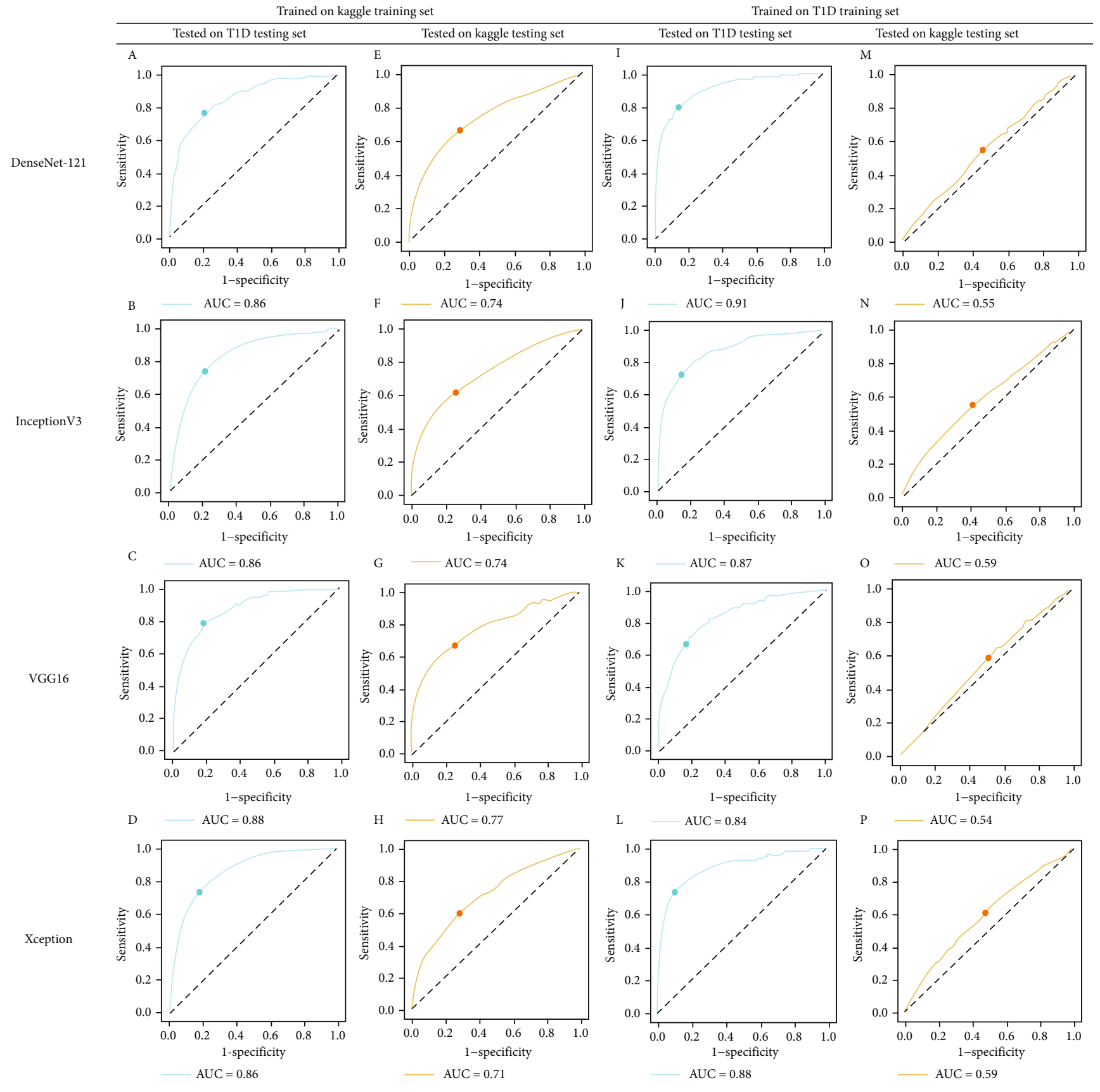

FIGURE 3: Receiver operating characteristic (ROC) curves of different transfer learning models in predicting diabetic retinopathy. The ROC curve of models that were tested with the type 1 diabetes (T1D) testing set was plotted in blue, whereas those tested with the Kaggle testing set were plotted in orange. The point on the ROC curve was the selected threshold. (e)-(h) There was a significant decrease in AUC when models previously trained with the T1D training set were tested with the Kaggle dataset. (i)-(l) The models that were previously trained with the Kaggle training set have a more robust performance when tested with the T1D testing set.

were then made using the final classifier. Class imbalance was addressed by estimating reweighting loss. Early stopping after 8-12 epochs of no improvement was applied to avoid overfitting, and the learning curves of both the training and validation sets were plotted to detect underfitting or overfitting. Binary cross entropy was used as the loss function, and stochastic gradient descent [23] or the Adam optimizer [24] was used with a learning rate of 1e-3 to 1e-4. Hyperparameters were optimized using random search. The development and analysis of the models were implemented using Keras 2.4.3 and Tensorflow 2.4.1 on Google colaboratory [25], whereas a part of image preprocessing and gradient-weighted class activation (Grad-CAM) visualization were run in Jupyter Notebook [26]. Two groups of models trained using the T1D and Kaggle training sets were tested in both the T1D and Kaggle testing sets (Figure 2).

In our model, CNNs including Inception-V3 [27], DenseNet-121 [28], VGG16 [29], and Xception [30] were 


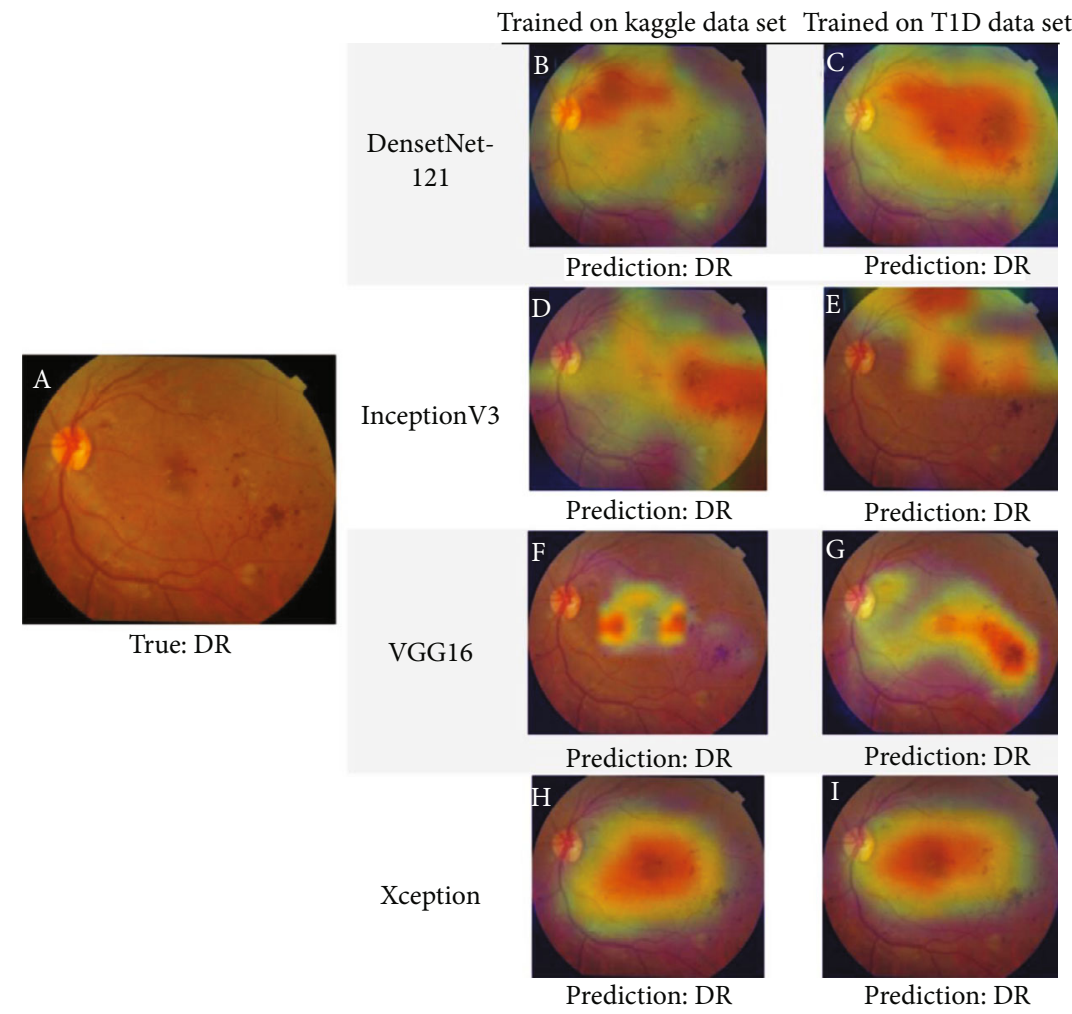

FIgURE 4: The images demonstrate the original (a) and superimposed Grad-CAM activation maps ((b)-(i)) of the selected diabetic retinopathy (DR) color fundus image. All models gave a true-positive prediction. There were some similarities in activation maps even in different transfer learning models trained with different datasets.

selected for model training because of their high performance in ImageNet Large Scale Visual Recognition Challenge and wide implementation in other medical image classifications [9]. All networks were pretrained on ImageNet [31]. Performance of the model with each CNN was evaluated.

2.5. Visualization Method. To observe how the models led to the prediction, the final convolutional layer of each model was extracted to obtain the activation map using the GradCAM visualization method [32], which highlighted the regions that provided an important contribution to the prediction. The activation map was then superimposed on the original image for interpretation.

2.6. Statistical Analysis. Receiver operating characteristic (ROC) curves were plotted using Matplotlib 3.2.2. Area under the ROC curve (AUC), sensitivity, and specificity were calculated to compare the performance of different models trained with both datasets using Python 3.7.1 and Sklearn 0.22.2. Optimal threshold of ROCs was chosen by maximizing the geometric mean of sensitivity and specificity. The descriptive results in this study are expressed as numbers and percentages for discrete variables.

\section{Results}

3.1. Image Characteristics. In the Kaggle dataset, 8,408 images were subsampled from the original dataset, with $6,150(73 \%)$ images classified as normal and 2,258 (27\%) images as DR. In the T1D dataset, 7,064 images from 475 patients with T1D were collected. Of these, 873 (13\%) images from 79 (17\%) patients were classified as DR.

3.2. Model Performance. Model performance is shown in Table 1. When the models were trained using the Kaggle imaging dataset, the overall AUC reached a mean (range) of $0.74(0.03)$ in the Kaggle testing set, with VGG16 providing the best performance $(\mathrm{AUC}=0.77)$. AUCs increased to a mean (range) of 0.87 (0.01) when the models trained with the Kaggle training set were tested using the T1D testing set. On the other hand, the transfer learning models achieved a mean (range) AUC of 0.88 (0.03) when trained and tested using the T1D imaging dataset, with DenseNet121 providing the best performance $(\mathrm{AUC}=0.91)$ and VGG16 the worst (AUC $=0.84)$. However, when models that were previously trained using the T1D training set were tested using the Kaggle dataset, AUCs significantly decreased to a mean (range) of 0.57 (0.02). The corresponding ROC curves are illustrated in Figure 3.

3.3. Class Activation Maps. The results of activation maps from different transfer learning models of both DR and normal cases are presented in Figures 4 and 5. Aside from highlighting the clinically observable retinal abnormalities, which were the traditional characteristic findings of DR, including microaneurysms, hemorrhages, and exudates (Figures 4(c), $4(\mathrm{~d})$, and $4(\mathrm{~g}))$, other regions including the macula (Figures 4(h), 4(i), 5(d), 5(h), 5(f), and 5(i)), optic disc 


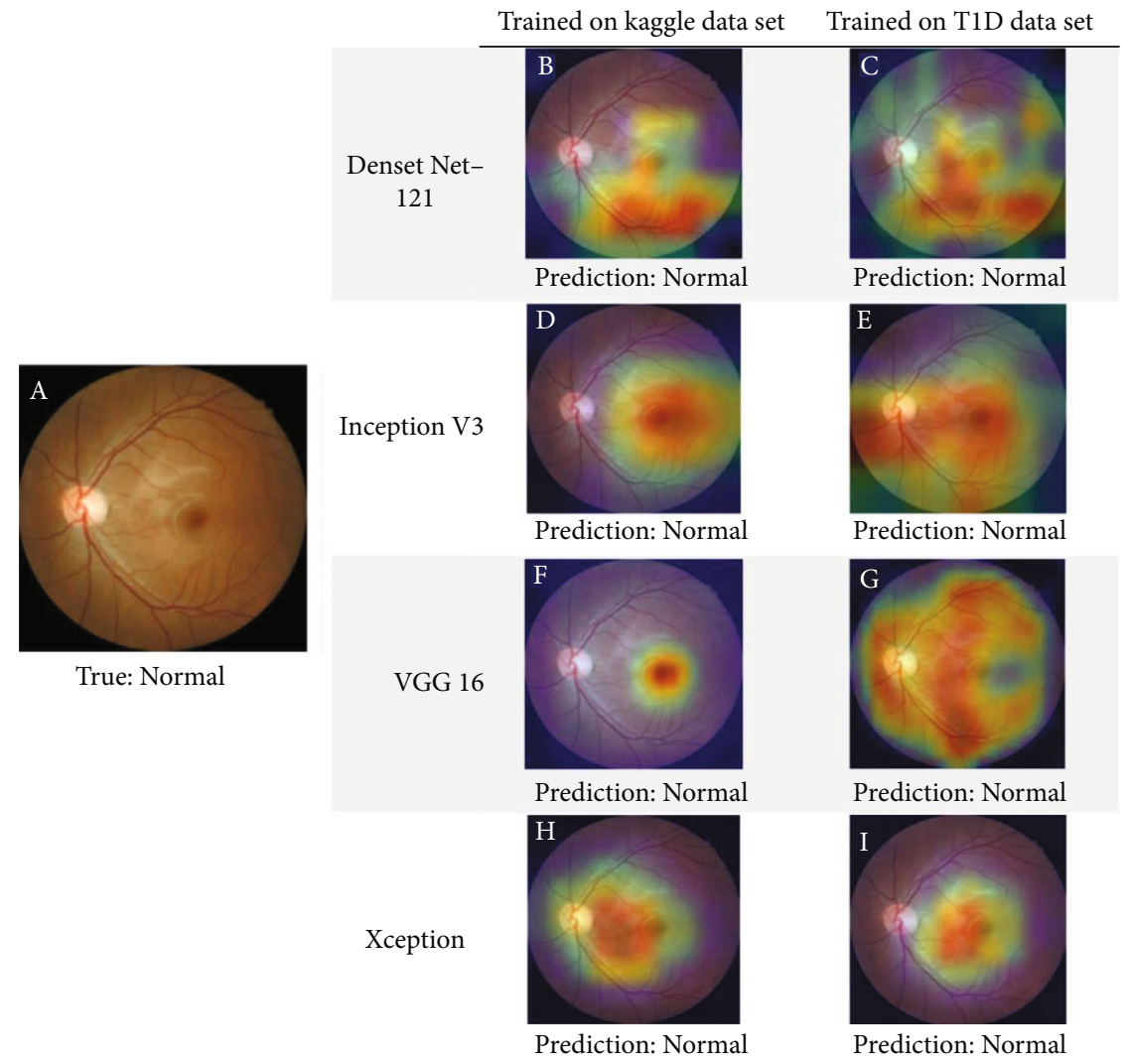

FIGURE 5: The images present the original (a) and superimposed Grad-CAM activation maps ((b)-(i)) of the selected normal color fundus image. All models gave a true-negative prediction. There was a high variation in the activation map when given a normal fundus image. Some models focus on the optic disc ((e) and (g)), whereas others highlight the retinal vessels ((b), (c), and (g)), or macular region ((d), (h), (f), and (i)).

(Figures 5(e) and 5(g)), and retinal vessels (Figures 5(b), $5(\mathrm{c})$, and $5(\mathrm{~g}))$ were also occasionally highlighted. Greater similarities were observed in activation maps among transfer learning models trained using the DR fundus image (Figure 4) rather than the normal fundus image (Figure 5).

\section{Discussion}

4.1. Main Findings of the Present Study. In our study, we trained models using the open-access Kaggle dataset, which has high image variability and theoretically predominant T2D patients, and the T1D dataset from the single medical center. We found that the model trained using the Kaggle dataset had an average AUC of 0.74 when testing using the same dataset, but this increased to 0.87 when testing using the T1D dataset. By contrast, the model trained using the T1D dataset had high accuracy (up to AUC of 0.91) in T1D patients, but it decreased (lowest AUC of 0.54) with the Kaggle dataset. Heatmaps demonstrated weighted features of retinal microaneurysm, hemorrhage, exudation, and vessels. Dataset homogeneity dataset may affect the trainability and generalization of the model.

4.2. Importance of External Validation and Standardization of Hyperparameters. Previous studies proposed numerous models that achieved high performance in diagnosing DR, even when trained with only a small dataset containing thousands of images [16]. The performance of our results yielded comparable results with the previous study (AUCs ranged from 0.65 to 0.86 ) when using a similar data size from the Kaggle dataset [33]. However, a large DR screening validation study found that most algorithms had significant performance differences and even obtained concerning results when evaluated through external validation, even though these algorithms were already in active use in realworld clinical settings [34]. In our study, models trained using the T1D dataset also exhibited acceptable performance (AUCs between 0.84 and 0.91 ) when internally validated, but their performance significantly decreased when evaluated using the external dataset. These results highlight the need for rigorous training and testing of models by using datasets containing a similar distribution of target population to avoid the huge discrepancy between expected and real performance. In addition, to produce a stable and reproducible prediction outcome, considerably more hyperparameters should be standardized. Although a previous study had already investigated the large number of possible factors that influence the performance of deep learning model [9], we anticipate that many more elements still need to be determined. For instance, the etiology of DM, age range, and comorbid eye diseases were shown to be possible influencing factors in our study. 


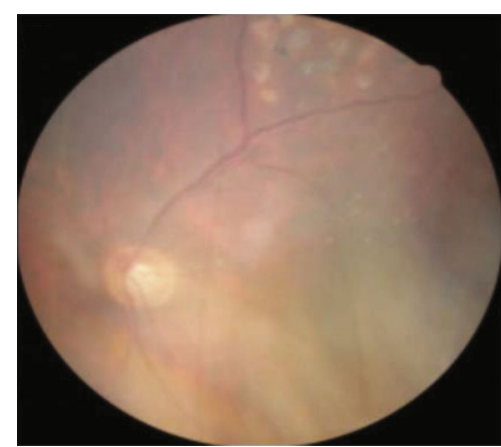

(a)

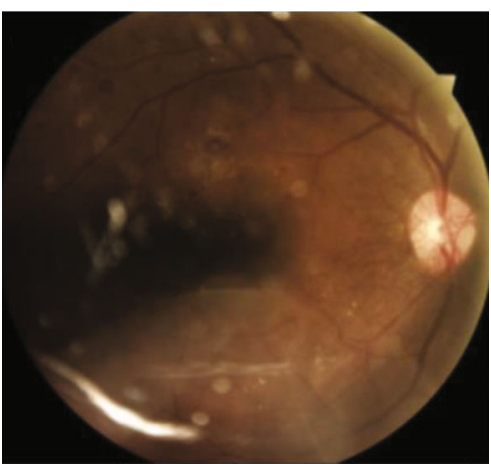

(c)

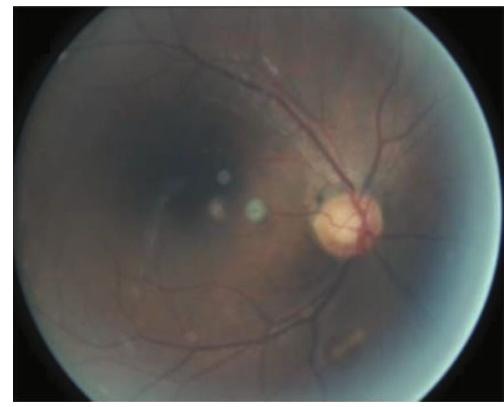

(e)

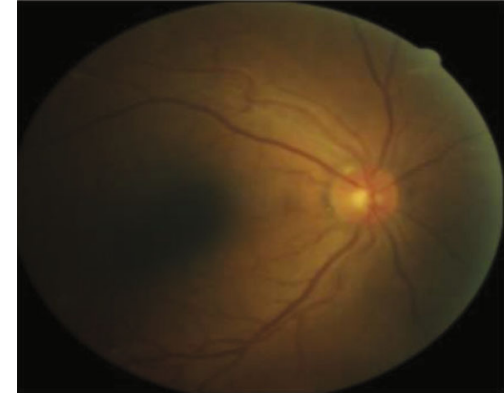

(b)

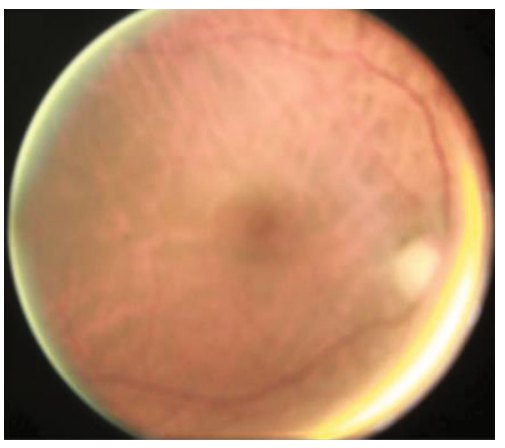

(d)

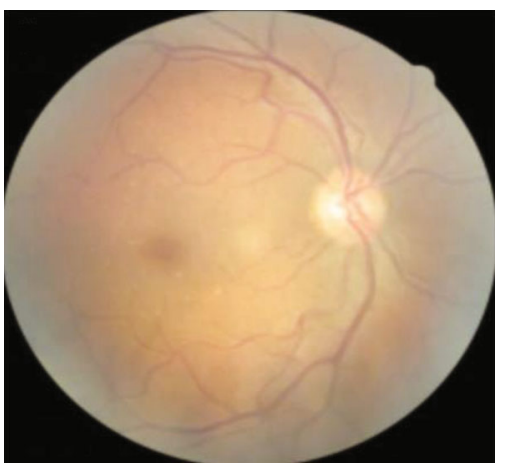

(f)

FIGURE 6: Images in the Kaggle dataset with wrong prediction. (a) False-negative in an image with foggy view and retinal laser scar. (b) Falsenegative in an image with poor illumination. (c) False-negative in an image with reflective spots and shadows. (d) False-positive in an image with overexposure and halo. (e) False-positive in an image with underexposure and halo. (f) False-positive in an image with exudates caused by age-related macular degeneration.

4.3. Different Performance Levels in Different Datasets. The models trained using the T1D dataset had poor performance when tested using the Kaggle dataset, whereas those trained using the Kaggle dataset showed better performance when tested using the T1D dataset. There are several possible explanations for the differences in the generalization of models trained using different datasets despite the use of the same method, similar dataset size, and imbalance ratio. When reviewing the wrong prediction images, we found that the images had similar problems affecting model prediction (Figure 6). Images from the T1D dataset were evaluated by retinal ophthalmologists, and images with poor quality or those that could not be graded were excluded. As the T1D dataset contained images of patients over multiple visits, the number of unique patients with T1D may be less than that in the Kaggle dataset, resulting in homogeneous data and thus rendering the T1D database much easier to predict for models trained using either of the databases. By contrast, images from the Kaggle dataset may not be cleaned and may contain more noise and artifacts, including out-of-focus, overexposure, or underexposure images, than images from the T1D dataset. Furthermore, the Kaggle dataset could be collected from a more diverse population with older age and higher age variation, thus, having more heterogeneous characteristics, whereas T1D patients had similar demographics and younger age. Therefore, patients in the Kaggle dataset may have other ocular diseases related to aging or other comorbidities such as cataract and age-related macular degeneration. Retinal features of ocular diseases other than DR, such as retinal exudates in age-related macular 
degeneration, may affect model prediction. In addition, cataracts may affect image quality. These findings have been reported in our previous studies $[35,36]$. As the T1D dataset contained images of patients over multiple visits, the characteristic variation in T1D may be less than that in the Kaggle dataset, also resulting in homogeneous data. Therefore, a homogeneous dataset may have resulted in higher trainability and lower generalization of models and vice versa in a more heterogenous dataset. Therefore, heterogeneity of the testing population also influences the performance of prediction models.

4.4. Highlighted Regions by Grad-CAM. Typical characteristic findings of DR, such as retinal microaneurysms, hemorrhages, exudates, and neovascularization, were among the most common highlighted regions by Grad-CAM in our study, consistent with a previous report [37]. In addition, nontraditional regions including the macula and optic disc were occasionally highlighted. As DR may also present with diabetic macular edema and neovascularization of the disc, abnormal features in these regions may also be extracted. Although neurodegeneration precedes vascular lesion in DR [38], whether deep learning models can detect abnormalities before the appearance of clinically observable lesions requires further investigation.

4.5. Limitations. This study has several limitations. First, we only assessed how training using only the T1D dataset affects detection performance; these results may not apply to other etiologies, such as the inherited form, maturity onset diabetes of the young, or other secondary causes. Second, our T1D data were collected from a single medical center and from a single ethnicity, making the dataset relatively small with less heterogeneity. Third, DR was identified using macula-centered retinal fundus images in the T1D dataset, instead of images obtained through 7 -field retinal fundus photography, as suggested by the Early Treatment Diabetic Retinopathy Study [39]. In addition, we did not justify further DR grading, which may help with the determination of treatment-required DR, because the detection of early $\mathrm{DR}$ in the T1D population could provide more information in patient care and education [18]. Finally, our models were developed with a limited combination of hyperparameters, and we did not conduct a combined model training on both datasets. A different implementation may thus provide different results.

\section{Conclusion}

Our study investigated a deep learning-based DR prediction model using two datasets. Our results showed that dataset homogeneity can have a significant effect on the trainability and generalization of the model. This implied that deep learning models should be trained with data similar to the target population and updated according to the landscape of DM to ensure a robust prediction and outcome. As the prevalence of diabetes continues to rise [2], along with an alarming increase in the frequency of T2D among youth [40], the epidemiology of diabetes will continue to change.
In addition, activation maps produced inferred that in addition to characteristic findings of DR, the macula and optic disc may also contribute to the detection of abnormalities in fundus imaging.

\section{Data Availability}

The T1D dataset is not publicly available due to the data security policy of Chang Gung Memorial Hospital and is available upon reasonable request.

\section{Disclosure}

The funding group had no role in study conduction and result interpretation.

\section{Conflicts of Interest}

The authors declare that there is no conflict of interest regarding the publication of this paper.

\section{Authors' Contributions}

Jui-En Lo and Eugene Yu-Chuan Kang contributed equally to this work.

\section{Acknowledgments}

The study was supported by the Chang Gung Memorial Hospital, Taiwan (CMRPG3K0481 and CMRPG3L0251).

\section{References}

[1] D. S. Fong, L. Aiello, T. W. Gardner et al., "Retinopathy in diabetes," Diabetes Care, vol. 27, Supplement 1, pp. S84-S87, 2004.

[2] C. J. Flaxel, R. A. Adelman, S. T. Bailey et al., "Diabetic retinopathy preferred practice pattern ${ }^{\circledR}$," Ophthalmology, vol. 127, pp. 66-145, 2020.

[3] American Diabetes Association, "Standards of medical care in diabetes," Diabetes Care, vol. 27, Supplement 1, pp. S15-S35, 2004.

[4] C.-F. Chou, C. E. Sherrod, X. Zhang et al., "Barriers to eye care among people aged 40 years and older with diagnosed diabetes, 2006-2010," Diabetes Care, vol. 37, pp. 180-188, 2014.

[5] V. Gulshan, L. Peng, M. Coram et al., "Development and validation of a deep learning algorithm for detection of diabetic retinopathy in retinal fundus photographs," JAMA, vol. 316, pp. 2402-2410, 2016.

[6] D. S. W. Ting, C. Y. Cheung, G. Lim et al., "Development and validation of a deep learning system for diabetic retinopathy and related eye diseases using retinal images from multiethnic populations with diabetes," JAMA, vol. 318, pp. 2211-2223, 2017.

[7] D. S. W. Ting, L. R. Pasquale, L. Peng et al., "Artificial intelligence and deep learning in ophthalmology," The British Journal of Ophthalmology, vol. 103, no. 2, pp. 167-175, 2019.

[8] M. M. Islam, H. C. Yang, T. N. Poly, W. S. Jian, and Y. C. Jack $\mathrm{Li}$, "Deep learning algorithms for detection of diabetic retinopathy in retinal fundus photographs: a systematic review and 
meta-analysis," Computer Methods and Programs in Biomedicine, vol. 191, p. 105320, 2020.

[9] M. Y. T. Yip, G. Lim, Z. W. Lim et al., "Technical and imaging factors influencing performance of deep learning systems for diabetic retinopathy," NPJ Digital Medicine, vol. 3, no. 1, p. 40, 2020.

[10] American Diabetes Association, "Diagnosis and classification of diabetes mellitus," Diabetes Care, vol. 33, Supplement_1, pp. S62-S69, 2010.

[11] S. Sivaprasad, B. Gupta, R. Crosby-Nwaobi, and J. Evans, "Prevalence of diabetic retinopathy in various ethnic groups: a worldwide perspective," Survey of Ophthalmology, vol. 57, pp. 347-370, 2012.

[12] Y.-D. Jiang, C.-H. Chang, T.-Y. Tai, J.-F. Chen, and L.M. Chuang, "Incidence and prevalence rates of diabetes mellitus in Taiwan: analysis of the 2000-2009 Nationwide Health Insurance database," Journal of the Formosan Medical Association, vol. 111, pp. 599-604, 2012.

[13] M. C. Eppens, M. E. Craig, J. Cusumano et al., "Prevalence of diabetes complications in adolescents with type 2 compared with type 1 diabetes," Diabetes Care, vol. 29, pp. 1300-1306, 2006.

[14] R. Klein, B. E. Klein, and S. E. Moss, "Visual impairment in diabetes," Ophthalmology, vol. 91, no. 1, pp. 1-9, 1984.

[15] S. Y. Wang, C. A. Andrews, W. H. Herman, T. W. Gardner, and J. D. Stein, "Incidence and risk factors for developing diabetic retinopathy among youths with type 1 or type 2 diabetes throughout the United States," Ophthalmology, vol. 124, pp. 424-430, 2017.

[16] M. T. Hagos and S. Kant, "Transfer learning based detection of diabetic retinopathy from small dataset," 2019, http://arxiv .org/abs/190507203.

[17] Kaggle, "Diabetic Retinopathy Detection," 2015, July 2012 https://www.kaggle.com/c/diabetic-retinopathy-detection.

[18] E. Y. Kang, F. S. Lo, J. P. Wang et al., "Nomogram for prediction of non-proliferative diabetic retinopathy in juvenile-onset type 1 diabetes: a cohort study in an Asian population," Scientific Reports, vol. 8, p. 12164, 2018.

[19] N. K. Wang, C. C. Lai, J. P. Wang et al., "Risk factors associated with the development of retinopathy $10 \mathrm{yr}$ after the diagnosis of juvenile-onset type 1 diabetes in Taiwan: a cohort study from the CGJDES," Pediatric Diabetes, vol. 17, pp. 407-416, 2016.

[20] K. G. Alberti and P. Z. Zimmet, "Definition, diagnosis and classification of diabetes mellitus and its complications. Part 1: diagnosis and classification of diabetes mellitus provisional report of a WHO consultation," Diabetic Medicine, vol. 15, pp. 539-553, 1998.

[21] C. P. Wilkinson, F. L. Ferris 3rd, R. E. Klein et al., "Proposed international clinical diabetic retinopathy and diabetic macular edema disease severity scales," Ophthalmology, vol. 110, pp. 1677-1682, 2003.

[22] F. Chollet, "Building powerful image classification models using very little data," The Keras Blog, 2016, July 2021 https://blog.keras.io/building-powerful-image-classificationmodels-using-very-little-data.html.

[23] S. Ruder, "An overview of gradient descent optimization algorithms," 2016, http://arxiv.org/abs/160904747.

[24] D. P. Kingma and J. Ba, "Adam: a method for stochastic optimization,” 2014, http://arxiv.org/abs/14126980.
[25] T. Carneiro, R. V. M. D. NóBrega, T. Nepomuceno, G. Bian, V. H. C. D. Albuquerque, and P. P. R. Filho, "Performance analysis of Google Colaboratory as a tool for accelerating deep learning applications," IEEE Access, vol. 6, pp. 61677-61685, 2018.

[26] T. Kluyver, B. Ragan-Kelley, F. Pérez et al., Jupyter Notebooks, A Publishing Format for Reproducible Computational Workflows, 2016.

[27] C. Szegedy, V. Vanhoucke, S. Ioffe, J. Shlens, and Z. B. Wojna, "Rethinking the Inception Architecture for Computer Vision," in Proceedings of the IEEE conference on computer vision and pattern recognition, pp. 2818-2826, 2016.

[28] G. Huang, Z. Liu, L. V. D. Maaten, and K. Q. Weinberger, "Densely connected convolutional networks," in 2017 IEEE Conference on Computer Vision and Pattern Recognition (CVPR), pp. 2261-2269, 2017.

[29] K. Simonyan and A. Zisserman, "Very deep convolutional networks for large-scale image recognition," 2014, http://arxiv .org/abs/14091556.

[30] F. Chollet, "Xception: deep learning with depthwise separable convolutions," in 2017 IEEE Conference on Computer Vision and Pattern Recognition (CVPR), pp. 1800-1807, 2017.

[31] O. Russakovsky, J. Deng, H. Su et al., "ImageNet large scale visual recognition challenge," International Journal of Computer Vision, vol. 115, no. 3, pp. 211-252, 2015.

[32] R. R. Selvaraju, M. Cogswell, A. Das, R. Vedantam, D. Parikh, and D. Batra, "Grad-cam: visual explanations from deep networks via gradient-based localization,” 2019, http://arxiv.org/ abs/1610.02391.

[33] P. Burlina, W. Paul, P. Mathew, N. Joshi, K. D. Pacheco, and N. M. Bressler, "Low-shot deep learning of diabetic retinopathy with potential applications to address artificial intelligence bias in retinal diagnostics and rare ophthalmic diseases," JAMA ophthalmology, vol. 138, no. 10, pp. 1070-1077, 2020.

[34] A. Y. Lee, R. T. Yanagihara, C. S. Lee et al., "Multicenter, headto-head, real-world validation study of seven automated artificial intelligence diabetic retinopathy screening systems," Diabetes Care, vol. 44, pp. 1168-1175, 2021.

[35] E. Y. Kang, L. Yeung, Y. L. Lee et al., "A multimodal imagingbased deep learning model for detecting treatment-requiring retinal vascular diseases: model development and validation study," JMIR Medical Informatics, vol. 9, no. 5, p. e28868, 2021.

[36] Y. T. Hsieh, L. M. Chuang, Y. D. Jiang et al., "Application of deep learning image assessment software VeriSee ${ }^{\mathrm{TM}}$ for diabetic retinopathy screening," Journal of the Formosan Medical Association, vol. 120, pp. 165-171, 2021.

[37] S. Keel, J. Wu, P. Y. Lee, J. Scheetz, and M. He, "Visualizing deep learning models for the detection of referable diabetic retinopathy and glaucoma," JAMA ophthalmology, vol. 137, no. 3, pp. 288-292, 2019.

[38] S. F. Abcouwer and T. W. Gardner, "Diabetic retinopathy: loss of neuroretinal adaptation to the diabetic metabolic environment," Annals of the New York Academy of Sciences, vol. 1311, pp. 174-190, 2014.

[39] G. H. Bresnick, D. B. Mukamel, J. C. Dickinson, and D. R. Cole, "A screening approach to the surveillance of patients with diabetes for the presence of vision-threatening retinopathy," Ophthalmology, vol. 107, pp. 19-24, 2000.

[40] J. N. Wei, F. C. Sung, C. C. Lin, R. S. Lin, C. C. Chiang, and L. M. Chuang, "National surveillance for type 2 diabetes mellitus in Taiwanese children," JAMA, vol. 290, no. 10, pp. 13451350, 2003. 